Gynäkologische Endokrinologie 2012·10:153-154 DOI 10.1007/s10304-012-0472-z

Online publiziert: 17. August 2012

(c) Springer-Verlag 2012

\title{
W. Urdl
}

Universitätsklinik für Frauenheilkunde und Geburtshilfe, Medizinische Universität Graz

\section{Endokrinologie der Schwangerschaft}

vom Synzytiotrophoblasten synthetisiert wird, spielt eine wichtige Rolle im Dialog zwischen Mutter und Kind. In der ersten Schwangerschaftshälfte hat diese Substanz wachstumsfördernde Wirkungen, wohingegen in der zweiten Schwangerschaftshälfte mittels hPL vorwiegend metabolische Vorgänge von Mutter und Ungeborenem gesteuert werden.

Mit dem Begriff der „fetoplazentaren Einheit" wird die Besonderheit der plazentaren Steroidbiosynthese beschrieben. Für die Bildung von $\mathrm{C}_{19}$-Steroiden (Androgene) und $\mathrm{C}_{18}$-Steroiden (Östrogene) fehlt der Plazenta die notwendige Enzymausstattung $\left(\mathrm{C}_{17}-\mathrm{C}_{20}\right.$-Lyase). Daher müssen die benötigten Vorstufen aus der mütterlichen und fetalen Nebennierenrinde [Dehydroepiandrosteronsulfat (DHEAS)] sowie der fetalen Leber (16a-DHEAS) zugeführt werden.

Des Weiteren sind in diesem Beitrag endokrine Mechanismen, so z. B. die Rolle des Kortikotropin-releasing-Hormons $(\mathrm{CRH})$, und neurohumorale Regulationen, die zu Beginn der Geburt und zur Zeit der Laktation entscheidend sind, umfassend dargestellt.

Im Beitrag von H. Juch und M. Gauster aus Graz mit dem Titel „Endokrinologie der humanen Plazenta“ werden zahlreiche endokrine und stoffwechselaktive Faktoren beschrieben, die in der Plazenta synthetisiert werden und offensichtlich dem Dialog zwischen Mutter und Kind dienen. Es ist bemerkenswert, dass bis dato die Physiologie bzw. Pathophysiologie bestimmter in der Plazenta nachweisbarer Substanzen nur teilweise geklärt werden konnte.

Die Schilddrüsenfunktion einer graviden Frau nimmt entscheidenden Einfluss auf den Verlauf der Schwangerschaft, das fetale Outcome und die intellektuelle und psychomotorische Entwicklung des Kindes. Andererseits beeinflusst auch die Schwangerschaft per se die Funktion der Schilddrüse, insbesondere durch die vermehrte Östrogenproduktion, die wiederum zu einem Anstieg des thyroxinbindenden Globulins und in der Folge zu einem Anstieg der Gesamtschilddrüsenhormonkonzentration führt, und durch den Einfluss von $\beta$-hCG, das eine dem thyroideastimulierenden Hormon (TSH) ähnliche Wirkung entfalten kann. Es ist daher entscheidend, laufende therapeutische Maßnahmen bei vorbestehenden Funktionsstörungen der Schilddrüse an die Bedürfnisse der Schwangerschaft anzupassen, wie auch bis zu Beginn der Gravidität unbekannte bzw. neu in der Schwangerschaft auftretende Hypo- oder Hyperthyreosen frühzeitig zu diagnostizieren und optimalerweise in interdisziplinärer Kooperation mit einem internistischen Endokrinologen zu behandeln. W. HungerBattefeld aus Jena beschreibt in ihrem Beitrag in übersichtlicher Weise aktuelle diagnostische und therapeutische Maßnahmen bei Funktionsstörungen der Schilddrüse in graviditate.

In einem weiteren interessanten Beitrag behandeln E. Grubinger, B. Böttcher, C. Brezinka und L. Wildt aus Innsbruck das Thema „Endokrine Erkrankungen in der Schwangerschaft". Während in der Gravidität Funktionsstörungen der Schilddrüse und das Auftreten eines Gestationsdiabetes relativ häufig sind, sind endokrine Erkrankungen wie

- eine Hyperprolaktinämie bei Prolaktinom, - das Cushing-Syndrom,
Auch das humane Plazentalaktogen (hPL), ein Proteohormon, das ebenfalls 
- die Akromegalie,

- Funktionsstörungen der Nebenschilddrüse,

- das adrenogenitale Syndrom,

- der M. Addison und

- das Sheehan-Syndrom

sehr selten, ja sogar echte Raritäten. Die Voraussetzung für einen möglichst komplikationslosen Schwangerschaftsverlauf und die Geburt eines gesunden Kindes bei Schwangeren mit derartigen Endokrinopathien ist deren frühzeitige Diagnose und adäquate Behandlung in Kooperation mit Vertretern anderer Fachdisziplinen.

S. Hutter und F. Kainer aus München befassen sich in ihrem Beitrag mit dem Gestationsdiabetes, einer Glukosetoleranzstörung, die 2010 in Deutschland bei $3,7 \%$ aller Schwangeren auftrat. In der Gravidität werden fließende Übergänge zwischen einer physiologischen Insulinresistenz und dem Krankheitsbild des Gestationsdiabetes beobachtet. Bei Letzterem liegt zusätzlich eine Insulinsekretionsstörung vor, die teilweise genetisch bedingt ist, aber auch durch sog. Lifestyle-Faktoren wie Übergewicht und Bewegungsmangel verursacht sein kann. Nichtbe- handelte maternale Hyperglykämien verursachen eine fetale Hyperglykämie mit konsekutiver Hyperinsulinämie und Makrosomie. Die Makrosomie ist mit einem erhöhten Risiko im Hinblick auf eine operative Entbindung und auf Geburtstraumata, z. B. Schulterdystokien, assoziiert. Ist der Zuckerhaushalt der Mutter nicht oder nur schlecht eingestellt, besteht für das Neugeborene die Gefahr von Hypoglykämien, Polyzythämie und Hyperbilirubinämie. Für diese Kinder besteht darüber hinaus ein erhöhtes Risiko, selbst im späteren Leben an einem Diabetes mellitus Typ 2 zu erkranken.

Die Diagnose eines Gestationsdiabetes basiert auf der Durchführung eines oralen Glukosetoleranztests, wobei entsprechende Richtlinien bei dessen Durchführung und Interpretation eingehalten werden müssen (in Risikofällen „Gelegenheitsglukosemessung “ vor der 24. Schwangerschaftswoche; Suchtest bei allen Schwangeren von der $24+0$. bis $27+6$. Schwangerschaftswoche; 2-stufiges Screening). Die Therapie richtet sich nach den erhobenen Blutzuckerwerten und nach fetalen Sonographieparametern (Dopplersonographie, Abdominalumfang $u$. a.).
Das seit Langem geforderte Screening aller Schwangeren auf einen Gestationsdiabetes wird durch die neue Mutterschaftsrichtlinie auch Teil des Leistungsumfangs der gesetzlichen Krankenkassen. In Österreich wurde der orale Glukosetoleranztest als Screeningmethode in den Mutter-Kind-Pass aufgenommen.

Ich wünsche Ihnen viele Anregungen bei der Lektüre!

Mit herzlichen kollegialen Grüßen

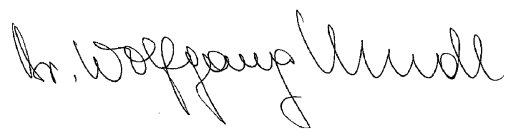

W. Urdl

\section{Korrespondenzadresse}

\section{Prof. Dr.W. Urdl}

Universitätsklinik für Frauenheilkunde und Geburtshilfe,

Medizinische Universität Graz

Kaiser-Franz-Josef-Kai 46

8010 Graz

wolfgang.urdl@ivf-institut.at

\section{Jahre GyNÄKOLOGISCHE ENDOKRINOLOGIE}

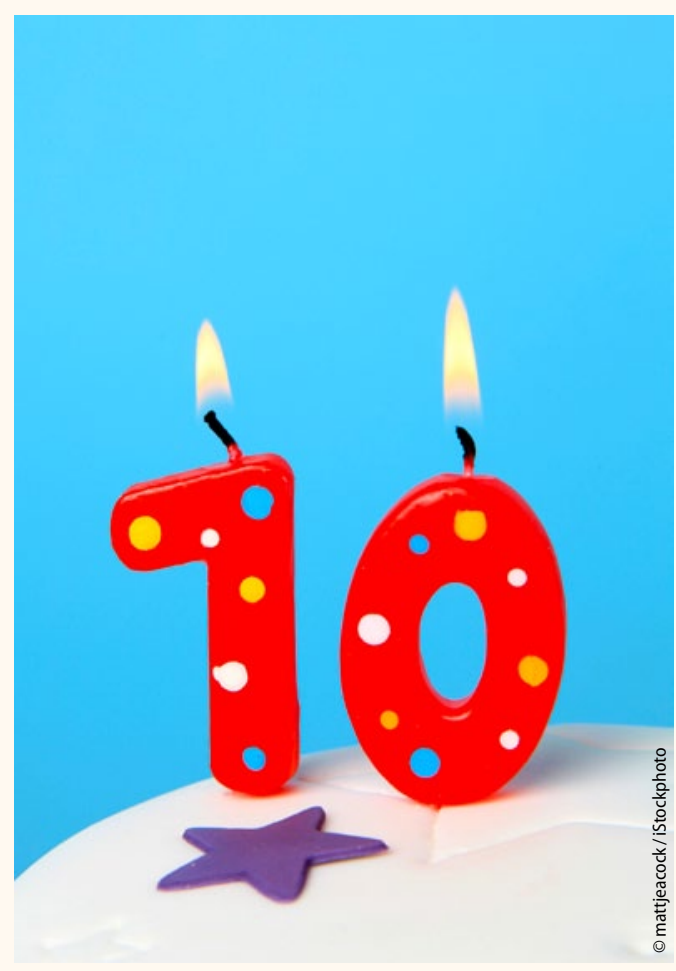

Liebe Leserinnen und Leser,

die GYNÄKOLOGISCHE ENDOKRINOLOGIE feiert Geburtstag!

Im Jahr 2002 wurde die Zeitschrift aus der Taufe gehoben mit dem Ziel, das umfassende Wissen um gynäkologisch-endokrinologische Prozesse mit einem „Blick über den Tellerrand“ zu vermitteln.

Wir danken unseren Herausgebern und Autoren, den Gutachtern und Ihnen, verehrte Leserinnen und Leser, für Ihr Interesse und Ihre Unterstützung. Gerne nehmen wir auch zukünftig Ihre Anregungen und Kritik für die GYNÄKOLOGISCHE ENDOKRINOLOGIE entgegen.

Auf weitere spannende Themen freut sich

Ihr Redaktionsteam 